\title{
THE MECHANICS OF BREATHING IN DIFFERENT BODY POSITIONS. II. IN CARDIOPULMONARY DISEASE
}

\author{
BY ERNST O. ATTINGER,1 J. AARON HERSCHFUS, AND MAURICE S. SEGAL \\ (From the Lung Station (Tufts) and Department of Inhalation Therapy, Boston City Hospital, \\ and the Department of Medicine, Tufts University School of Medicine, Boston, Mass.)
}

(Submitted for publication July 8, 1955; accepted April 16, 1956)

In a previous paper, the effects of changes in body position upon the mechanics of breathing in normals were reported (1). This report deals with the results obtained in two groups of patients studied in the supine, sitting, prone, head down and lateral positions.

\section{MATERIAL AND METHODS}

The technic described in the previous paper has been employed throughout the study. It consists essentially of measuring intraesophageal pressures, air flow rates and volume changes simultaneously and continuously throughout the respiratory cycle. From these measurements, compliance (volume change per unit pressure change expressed as $\mathrm{L}$. per $\mathrm{cm} . \mathrm{H}_{2} \mathrm{O}$ ) and mechanical resistance (pressure necessary to obtain a given flow rate, expressed in $\mathrm{cm}$. per $\mathrm{L}$. per sec.) have been determined in the different body positions and at different respiratory rates. Mechanical resistance is reported as the mean mechanical resistance of the inspiratory and expiratory phases of the respiratory cycle. In addition, vital capacities were determined with a Benedict-Roth type spirometer, maximal breathing capacities with a low resistance Rudolph valve and a Douglas bag during a 15 -second period, and residual volume and intrapulmonary mixing by Darling's seven-minute nitrogen washout method. These tests were carried out in the sitting position. Arterial blood gases, obtained from the brachial artery, were determined with the van Slyke technic, $\mathrm{pH}$ with a Cambridge Research Model $\mathrm{pH}$ Meter and $\mathrm{pCO}_{2}$ by Hasting and Singer's Nomogram.

The first group of patients consisted of twelve subjects whose predominant disease was chronic pulmonary emphysema. The second group was composed of various cardiopulmonary diseases. Each patient was studied in at least two positions at slow and rapid respiratory rates. The rates were selected according to the condition of the patient. The physical characteristics of the patients together with their diagnosis and the results of routine pulmonary function tests appear in Tables Ia and Ib. Patients M. N. and H. P. were studied on two separate occasions during different degrees of disability.

\footnotetext{
1 Research Fellow in Medicine, Tufts University School of Medicine.
}

\section{RESULTS $^{2}$}

The results are presented in Tables II, III, IV and $\mathrm{V}$.

1. Patients with chronic pulmonary emphysema studied in the supine, sitting and prone positions

The end expiratory pressures are as a rule highest in the supine position and lowest in the sitting position. However, because of the considerable variation of the individual results, the difference of the means is not statistically significant.

Compliance values are lower in the supine and sitting positions as compared to the previously reported normal group whereas the values for the prone position are generally higher. With the exception of the prone position, the differences of the means are significant at the 1 per cent level. The compliance is lowest in the supine position, rises considerably in the sitting position and remains high in the prone position. These differences are significant at the 5 per cent level. In all positions, there is a marked decrease of compliance with increase in the respiratory rate; the greatest drop occurs in the supine position.

Mechanical resistance is significantly higher during expiration than during inspiration in all positions and at all respiratory rates. Both expiratory and inspiratory resistances are highest in the supine position and show a considerable drop in the sitting position. The values in the prone position are usually between these two extremes. There is no significant difference between the resistance values in a given position at different respiratory rates. As compared to the normal group, inspiratory resistance is, on the average, in-

2 The statistical analysis has been carried out by the methods described in the first paper (1). 
TABLE Ia

Patients with chronic pulmonary emphysema

\begin{tabular}{|c|c|c|c|c|c|c|c|c|c|c|c|}
\hline \multirow[b]{2}{*}{ Patient } & \multirow[b]{2}{*}{ Diagnosis } & \multirow[b]{2}{*}{ Sex } & \multirow[b]{2}{*}{ Age } & \multirow[b]{2}{*}{$\underset{\left(m^{2}\right)}{\text { B.S. }}$} & \multirow[b]{2}{*}{$\underset{(\dot{m} l .)}{\text { V.C. }}$} & \multirow[b]{2}{*}{ (L./min.) } & \multirow[b]{2}{*}{$\underset{(\%)}{\mathrm{RV} / \mathrm{TC}}$} & \multirow{2}{*}{$\begin{array}{c}\text { Intra- } \\
\text { pulmonary } \\
\text { mixing } \\
\left(N_{2} \%\right)\end{array}$} & \multicolumn{3}{|c|}{ Arterial blood } \\
\hline & & & & & & & & & $\mathrm{pH}$ & $\begin{array}{l}\mathrm{O}_{2} \text { sat. } \\
(\%)\end{array}$ & $\mathrm{pCO}_{2}$ \\
\hline N.S. & $\begin{array}{l}\text { Chronic pulmonary } \\
\text { emphysema, secondary } \\
\text { polycythemia }\end{array}$ & $\mathbf{M}$ & 64 & 1.85 & 3,000 & & & & 7.39 & 85.2 & 54.0 \\
\hline A. A. & $\begin{array}{l}\text { Chronic pulmonary } \\
\text { emphysema }\end{array}$ & $\mathbf{M}$ & 59 & 1.70 & 2,000 & 54.0 & & & 7.39 & 84.3 & 40.0 \\
\hline W. L. & $\begin{array}{l}\text { Chronic pulmonary } \\
\text { emphysema }\end{array}$ & $\mathbf{M}$ & 49 & 1.99 & 1,900 & & 65 & 13.0 & 7.42 & 76.5 & 50.0 \\
\hline J.S. & $\begin{array}{l}\text { Chronic pulmonary } \\
\text { emphysema, hyper- } \\
\text { tension }\end{array}$ & $\mathbf{M}$ & 55 & 1.77 & 3,410 & 61.0 & 53 & 7.75 & 7.41 & 83.9 & 44.0 \\
\hline M. P. & $\begin{array}{l}\text { Chronic pulmonary } \\
\text { emphysema, respira- } \\
\text { tory acidosis }\end{array}$ & $\mathbf{M}$ & 64 & 1.72 & 3,000 & 49.0 & & & 7.28 & 67.7 & 60.0 \\
\hline A. $R$. & $\begin{array}{l}\text { Chronic pulmonary } \\
\text { emphysema, cor } \\
\text { pulmonale }\end{array}$ & $\mathbf{M}$ & 61 & 1.53 & 3,320 & 32.0 & 57 & 14.1 & 7.40 & 86.5 & 43.0 \\
\hline W. G. & $\begin{array}{l}\text { Chronic pulmonary } \\
\text { emphysema, cor } \\
\text { pulmonale }\end{array}$ & $\mathbf{M}$ & 61 & 2.07 & 2,690 & 49.6 & 57 & 3.1 & 7.39 & 88.5 & 35.5 \\
\hline A. B. & $\begin{array}{l}\text { Chronic pulmonary } \\
\text { emphysema, kyphosco- } \\
\text { liosis, polycythemia }\end{array}$ & $\mathbf{M}$ & 61 & 1.76 & 3,170 & 100.0 & 46 & 6.0 & 7.40 & 85.7 & 43.0 \\
\hline$\underset{(I)}{\text { M. N. }}$ & $\begin{array}{l}\text { Chronic pulmonary } \\
\text { emphysema, chronic } \\
\text { bronchitis }\end{array}$ & $\mathbf{M}$ & 50 & 1.68 & 2,955 & 51.2 & 64 & 9.7 & 7.40 & 86.0 & 44.0 \\
\hline$\underset{\text { (II) }}{\text { M. }} \mathbf{N}$. & Same as above & $\mathbf{M}$ & 50 & 1.68 & 2,200 & 47.0 & & & & & \\
\hline M. S. & $\begin{array}{l}\text { Chronic pulmonary } \\
\text { emphysema }\end{array}$ & $\mathbf{M}$ & 48 & 1.74 & 4,230 & 115.0 & 39 & 2.2 & & & \\
\hline J.S. & $\begin{array}{l}\text { Chronic pulmonary } \\
\text { emphysema }\end{array}$ & $\mathbf{M}$ & 48 & 1.61 & 2,340 & 50.0 & 48 & 11.7 & 7.40 & 87.7 & 44.0 \\
\hline
\end{tabular}

TABLE Ib

Patients with various cardiopulmonary diseases

\begin{tabular}{|c|c|c|c|c|c|c|c|c|c|c|c|}
\hline \multirow[b]{2}{*}{ Patient } & \multirow[b]{2}{*}{ Diagnosis } & \multirow[b]{2}{*}{ Sex } & \multirow[b]{2}{*}{ Age } & \multirow[b]{2}{*}{$\underset{\left(m^{2}\right)}{\text { B.S. }}$} & \multirow[b]{2}{*}{$\underset{(m l .)}{\text { V.C. }}$} & \multirow[b]{2}{*}{ M.B.C. } & \multirow[b]{2}{*}{ RV/\%C } & \multirow{2}{*}{$\begin{array}{l}\text { Intra- } \\
\text { pulmonary } \\
\text { mixing } \\
\left(N_{2} \%\right)\end{array}$} & \multicolumn{3}{|c|}{ Arterial blood } \\
\hline & & & & & & & & & pH & $\begin{array}{l}\mathrm{O}_{2} \text { sat. } \\
(\%)\end{array}$ & $\mathrm{pCO}_{2}$ \\
\hline R. W. & $\begin{array}{l}\text { Chronic bronchial } \\
\text { asthma }\end{array}$ & $\mathbf{M}$ & 35 & 1.32 & 4,400 & & & & & & \\
\hline$\underset{(I)}{H . P .}$ & $\begin{array}{l}\text { Chronic bronchial } \\
\text { asthma, chronic } \\
\text { pulmonary emphysema }\end{array}$ & $\mathbf{M}$ & 16 & 1.85 & 3,600 & 114.0 & & & 7.37 & 94.5 & 41.5 \\
\hline$\underset{\text { (II) }}{\mathrm{H}} . \mathrm{P}$. & Same as above & $\mathbf{M}$ & 16 & 1.85 & 2,500 & 73.0 & 60 & 6.0 & & & \\
\hline M. G. & $\begin{array}{l}\text { Chronic bronchial } \\
\text { asthma, chronic } \\
\text { pulmonary emphysema }\end{array}$ & $\mathbf{F}$ & 31 & 1.82 & 2,700 & 43.3 & 47.4 & 3.8 & 7.40 & 92.0 & 35.0 \\
\hline $\begin{array}{l}\text { A. DeW. } \\
\text { B. B. }\end{array}$ & $\begin{array}{l}\text { Scleroderma } \\
\text { Left pneumonectomy for } \\
\text { bronchial carcinoma }\end{array}$ & $\begin{array}{l}\mathbf{F} \\
\mathbf{M}\end{array}$ & $\begin{array}{l}51 \\
65\end{array}$ & $\begin{array}{l}1.56 \\
1.67\end{array}$ & $\begin{array}{l}2,300 \\
2,500\end{array}$ & $\begin{array}{l}58.0 \\
35.0\end{array}$ & $\begin{array}{l}49 \\
50\end{array}$ & $\begin{array}{r}6.2 \\
13.5\end{array}$ & $\begin{array}{l}7.42 \\
7.38\end{array}$ & $\begin{array}{l}97.2 \\
82.1\end{array}$ & $\begin{array}{l}39.0 \\
50.0\end{array}$ \\
\hline E. J. & $\begin{array}{l}\text { Aneurysm of aorta with } \\
\text { atelectasis of left lung }\end{array}$ & $\mathbf{M}$ & 63 & 1.72 & 1,530 & 52.0 & 56 & 19.6 & 7.41 & 81.4 & 39.0 \\
\hline $\begin{array}{l}\text { W. F. } \\
\text { G. A. } \\
\text { A. A. }\end{array}$ & $\begin{array}{l}\text { Calcified right pleura } \\
\text { Pleurisy left } \\
\text { Cardiac failure, pleural } \\
\text { effusion right }\end{array}$ & $\begin{array}{l}\mathbf{M} \\
\mathbf{M}\end{array}$ & $\begin{array}{l}73 \\
56 \\
65\end{array}$ & $\begin{array}{l}1.56 \\
1.97 \\
1.53\end{array}$ & $\begin{array}{l}2,760 \\
2,700 \\
2,500\end{array}$ & $\begin{array}{r}66.0 \\
114.0 \\
46.7\end{array}$ & 47 & 3.46 & $\begin{array}{l}7.39 \\
7.37\end{array}$ & $\begin{array}{l}88.6 \\
94.3 \\
92.5\end{array}$ & $\begin{array}{l}37.0 \\
40.5\end{array}$ \\
\hline J. Q. & Mitral stenosis & $\mathbf{M}$ & 23 & 1.76 & 4,700 & 132.2 & & & 7.39 & 98.1 & 39.0 \\
\hline
\end{tabular}


TABLE II

Mechanics of breathing: Results in twelve patients with chronic pulmonary emphysema in supine, sitting and prone positions during slow and fast respiratory rates

\begin{tabular}{|c|c|c|c|c|c|c|c|c|c|c|c|c|}
\hline \multirow[b]{3}{*}{ Patient } & \multicolumn{6}{|c|}{ Respiratory rate } & \multicolumn{6}{|c|}{$\begin{array}{l}\text { Tidal volume } \\
(m l .)\end{array}$} \\
\hline & \multicolumn{2}{|c|}{ Supine } & \multicolumn{2}{|c|}{ Sitting } & \multicolumn{2}{|c|}{ Prone } & \multicolumn{2}{|c|}{ Supine } & \multicolumn{2}{|c|}{ Sitting } & \multicolumn{2}{|c|}{ Prone } \\
\hline & SR & $\mathbf{F R}$ & SR & $\overline{F R}$ & $\mathbf{S R}$ & $\mathbf{F R}$ & SR & $\mathbf{F R}$ & SR & $\mathbf{F R}$ & SR & $\overline{F R}$ \\
\hline $\begin{array}{l}\text { N.S. } \\
\text { A. A. } \\
\text { W. L. } \\
\text { J.S. } \\
\text { M.P. } \\
\text { A. R. } \\
\text { W.G. } \\
\text { A. B. } \\
\text { M. N. (I)* } \\
\text { M. N. (II)† } \\
\text { M.S. } \\
\text { J.S. }\end{array}$ & $\begin{array}{l}24 \\
15 \\
15 \\
27 \\
22 \\
15 \\
20 \\
17 \\
18 \\
18 \\
23 \\
17\end{array}$ & $\begin{array}{r}40 \\
33 \\
100 \\
60 \\
55 \\
55 \\
85 \\
75 \\
95 \\
125 \\
80\end{array}$ & $\begin{array}{l}24 \\
18 \\
17 \\
27 \\
22 \\
20 \\
22 \\
24 \\
20 \\
16 \\
24 \\
24\end{array}$ & $\begin{array}{r}30 \\
95 \\
75 \\
36 \\
80 \\
75 \\
75 \\
80 \\
185 \\
68\end{array}$ & $\begin{array}{l}30 \\
22 \\
20 \\
27 \\
22 \\
22 \\
18 \\
\\
24\end{array}$ & $\begin{array}{r}100 \\
75 \\
40 \\
76 \\
54 \\
75 \\
94 \\
\\
80\end{array}$ & $\begin{array}{l}460 \\
460 \\
550 \\
486 \\
700 \\
630 \\
770 \\
650 \\
550 \\
730 \\
460 \\
430\end{array}$ & $\begin{array}{l}840 \\
360 \\
150 \\
370 \\
280 \\
550 \\
510 \\
285 \\
325 \\
900 \\
320\end{array}$ & $\begin{array}{l}520 \\
560 \\
615 \\
600 \\
775 \\
490 \\
770 \\
615 \\
565 \\
876 \\
450 \\
520\end{array}$ & $\begin{array}{l}490 \\
200 \\
400 \\
350 \\
420 \\
530 \\
335 \\
454 \\
870 \\
380\end{array}$ & $\begin{array}{r}585 \\
875 \\
705 \\
1,060 \\
675 \\
530 \\
800 \\
500\end{array}$ & $\begin{array}{l}220 \\
300 \\
390 \\
540 \\
590 \\
350 \\
400 \\
\\
290\end{array}$ \\
\hline \multirow[t]{3}{*}{ Mean } & 19.3 & 73 & 21.5 & 80 & 23 & 74 & 575 & 445 & 615 & 465 & 715 & 385 \\
\hline & \multicolumn{6}{|c|}{$\begin{array}{l}\text { Intraesophageal end expiratory pressure } \\
\qquad(\mathrm{cm} . \mathrm{H}: 0)\end{array}$} & \multicolumn{6}{|c|}{$\begin{array}{l}\text { Compliance } \\
\left(\mathrm{L} . / \mathrm{cm} . \mathrm{H} \mathrm{S}_{\mathrm{O}}\right)\end{array}$} \\
\hline & \multicolumn{2}{|c|}{ Supine } & \multicolumn{2}{|c|}{ Sitting } & \multicolumn{2}{|c|}{ Prone } & \multicolumn{2}{|c|}{ Supine } & \multicolumn{2}{|c|}{ Sitting } & \multicolumn{2}{|c|}{ Prone } \\
\hline Patient & $\mathbf{S R}$ & FR & $\mathbf{S R}$ & FR & SR & $\mathbf{F R}$ & SR & $\mathbf{F R}$ & SR & FR & SR & FR \\
\hline $\begin{array}{l}\text { N. S. } \\
\text { A. A. } \\
\text { W. L. } \\
\text { J.S. } \\
\text { M. P. } \\
\text { A. R. } \\
\text { W. G. } \\
\text { A. B. } \\
\text { M. N. (I)* } \\
\text { M. N. (II)† } \\
\text { M. S. } \\
\text { J.S. }\end{array}$ & $\begin{array}{c}-2.0 \\
-1.5 \\
0 \\
-5.4 \\
-0.5 \\
+0.2 \\
-0.4 \\
-1.6 \\
-3.2 \\
+0.2 \\
+0.25 \\
-2.0\end{array}$ & $\begin{array}{l}-0.7 \\
+4.4 \\
-4.0 \\
-3.0 \\
-0.1 \\
-0.6 \\
-4.5 \\
-2.3 \\
-7.5 \\
+3.0 \\
+2.7\end{array}$ & $\begin{array}{l}-4.5 \\
-1.2 \\
-7.0 \\
-2.2 \\
-4.0 \\
-2.0 \\
-2.7 \\
-5.0 \\
-4.8 \\
-1.75 \\
-6.0 \\
+2.0\end{array}$ & $\begin{array}{c}-7.0 \\
-3.0 \\
-5.0 \\
-0.4 \\
-3.3 \\
-4.4 \\
-5.4 \\
5 \\
-2.5 \\
-0.2\end{array}$ & $\begin{array}{c}-6.9 \\
-6.5 \\
0 \\
-0.3 \\
-6.3 \\
-4.0 \\
-0.2 \\
-2.0\end{array}$ & $\begin{array}{l}-4.6 \\
5-7.0 \\
-2.1 \\
+0.8 \\
3-7.0 \\
-4.5 \\
+1.7 \\
0-3.8\end{array}$ & $\begin{array}{l}0.072 \\
0.110 \\
0.029 \\
0.072 \\
0.148 \\
0.139 \\
0.132 \\
0.092 \\
0.124 \\
0.111 \\
0.118 \\
0.067\end{array}$ & $\begin{array}{l}0.064 \\
\\
0.018 \\
0.022 \\
0.090 \\
0.050 \\
0.095 \\
0.072 \\
0.078 \\
0.040 \\
0.065 \\
0.025\end{array}$ & $\begin{array}{l}0.110 \\
0.120 \\
0.075 \\
0.134 \\
0.270 \\
0.195 \\
0.170 \\
0.100 \\
0.215 \\
0.186 \\
0.196 \\
0.110\end{array}$ & $\begin{array}{l}0.034 \\
0.061 \\
0.155 \\
0.110 \\
0.154 \\
0.092 \\
0.165 \\
0.062 \\
0.066 \\
0.064\end{array}$ & $\begin{array}{l}0.134 \\
0.270 \\
0.233 \\
0.235 \\
0.090 \\
0.262 \\
0.158 \\
\\
0.068\end{array}$ & $\begin{array}{l}0.078 \\
0.180 \\
0.163 \\
0.088 \\
0.081 \\
0.173 \\
0.052 \\
0.044\end{array}$ \\
\hline \multirow[t]{4}{*}{ Mean } & -1.75 & -0.5 & -3.3 & -2.9 & -3.3 & $\begin{array}{ll}3 & -3.32\end{array}$ & 0.101 & 0.056 & 0.157 & 0.096 & 0.181 & 0.108 \\
\hline & \multicolumn{6}{|c|}{$\begin{array}{l}\text { Mean mechanical resistance during inspiration } \\
\left(\mathrm{cm} . \mathrm{H}_{2} \mathrm{O} / \mathrm{L} . / \mathrm{sec} .\right)\end{array}$} & \multicolumn{6}{|c|}{$\begin{array}{c}\text { Mean mechanical resistance during expiration } \\
(\mathrm{cm} . \mathrm{H} \mathrm{O} / \mathrm{L} . / \mathrm{sec} .)\end{array}$} \\
\hline & \multicolumn{2}{|c|}{ Supine } & \multicolumn{2}{|c|}{ Sitting } & \multicolumn{2}{|c|}{ Prone } & \multicolumn{2}{|c|}{ Supine } & \multicolumn{2}{|c|}{ Sitting } & \multicolumn{2}{|c|}{ Prone } \\
\hline & SR & FR & SR & FR & SR & FR & SR & FR & SR & FR & SR & FR \\
\hline $\begin{array}{l}\text { N. S. } \\
\text { A. A. } \\
\text { W. L. } \\
\text { J.S. } \\
\text { M. P. } \\
\text { A. R. } \\
\text { W. G. } \\
\text { A. B. } \\
\text { M. N. (I)* } \\
\text { M. N. (II)† } \\
\text { M. S. } \\
\text { J.S. }\end{array}$ & $\begin{array}{r}9.00 \\
6.95 \\
38.80 \\
10.10 \\
6.80 \\
10.20 \\
9.05 \\
5.33 \\
9.36 \\
6.60 \\
4.20 \\
8.00\end{array}$ & \begin{tabular}{r|}
6.10 \\
\\
29.00 \\
10.30 \\
10.70 \\
8.90 \\
7.20 \\
3.48 \\
8.75 \\
5.65 \\
3.10 \\
8.25
\end{tabular} & $\begin{array}{r}5.10 \\
4.35 \\
16.60 \\
4.90 \\
7.30 \\
5.20 \\
7.60 \\
3.22 \\
4.90 \\
6.70 \\
2.20 \\
4.75\end{array}$ & $\begin{array}{r}24.00 \\
4.00 \\
7.50 \\
6.36 \\
6.95 \\
2.86 \\
3.60 \\
3.45 \\
2.16 \\
3.65\end{array}$ & $\begin{array}{r}3.65 \\
7.90 \\
10.50 \\
5.40 \\
2.43 \\
4.40 \\
5.65 \\
\\
5.95\end{array}$ & $\begin{array}{r}3.85 \\
12.00 \\
7.30 \\
4.95 \\
2.23 \\
4.40 \\
3.45 \\
\\
6.20\end{array}$ & $\begin{array}{r}21.20 \\
7.85 \\
41.75 \\
48.00 \\
13.40 \\
15.15 \\
9.00 \\
9.60 \\
13.80 \\
9.50 \\
3.85 \\
8.00\end{array}$ & $\begin{array}{r}8.30 \\
\\
76.00 \\
27.00 \\
15.85 \\
14.50 \\
20.00 \\
4.90 \\
8.40 \\
7.75 \\
5.60 \\
17.80\end{array}$ & $\begin{array}{r}4.67 \\
6.10 \\
34.20 \\
11.78 \\
10.00 \\
13.90 \\
10.90 \\
4.11 \\
8.80 \\
6.00 \\
2.10 \\
3.20\end{array}$ & $\begin{array}{r}31.50 \\
12.20 \\
14.20 \\
11.55 \\
11.90 \\
2.94 \\
4.55 \\
7.23 \\
2.25 \\
5.40\end{array}$ & $\begin{array}{r}9.00 \\
14.70 \\
19.65 \\
5.45 \\
5.19 \\
6.44 \\
6.45 \\
\\
6.70\end{array}$ & $\begin{array}{r}7.77 \\
16.90 \\
11.10 \\
3.70 \\
3.35 \\
5.65 \\
5.55 \\
\\
\\
16.00\end{array}$ \\
\hline Mean & 10.40 & 9.20 & 6.05 & 6.45 & 5.75 & 5.55 & 16.80 & 18.75 & 9.65 & 10.40 & 9.20 & 8.75 \\
\hline
\end{tabular}

* First study.

† Second study-two months later. 
creased three times and expiratory resistance five times (significant at 0.1 per cent level). Similar differences between normal subjects and patients with chronic pulmonary emphysema have been reported by others $(2-6)$.
2. Patients with various cardiovascular diseases (studied in supine, sitting and prone positions)

Compliance values are significantly lower and resistance values significantly higher than in the normal group. Even though this group covers a

TABLE III

Mechanics of breathing: Results in patients with various cardiovascular diseases in supine, sitting and prone positions during slow and fast respiratory rates

\begin{tabular}{|c|c|c|c|c|c|c|c|c|c|c|c|}
\hline \multirow[b]{3}{*}{ Patient } & \multicolumn{5}{|c|}{ Respiratory rate } & \multicolumn{6}{|c|}{$\begin{array}{l}\text { Tidal volume } \\
(m l .)\end{array}$} \\
\hline & \multicolumn{2}{|c|}{ Supine } & \multicolumn{2}{|c|}{ Sitting } & Prone & \multicolumn{2}{|c|}{ Supine } & \multicolumn{2}{|c|}{ Sitting } & \multicolumn{2}{|c|}{ Prone } \\
\hline & SR & FR & $\mathbf{S R}$ & $\mathbf{F R}$ & FR & SR & FR & SR & $\mathbf{F R}$ & SR & $\mathbf{F R}$ \\
\hline $\begin{array}{l}\text { R. W. } \\
\text { A. DeW. } \\
\text { E. J. } \\
\text { B. B. } \\
\text { W. F. } \\
\text { G. A. } \\
\text { H. P. (I)* } \\
\text { H. P. (II)† } \\
\text { M. G. } \\
\text { J. Q. }\end{array}$ & $\begin{array}{l}15 \\
20 \\
30 \\
20 \\
16 \\
11 \\
17 \\
22 \\
11 \\
10\end{array}$ & $\begin{array}{r}68 \\
94 \\
43 \\
50 \\
65 \\
80 \\
90 \\
100 \\
55 \\
65\end{array}$ & $\begin{array}{l}13 \\
17 \\
25 \\
30 \\
17 \\
14 \\
18 \\
20 \\
11 \\
15\end{array}$ & $\begin{array}{r}60 \\
107 \\
75 \\
70 \\
71 \\
80 \\
80 \\
115 \\
79 \\
60\end{array}$ & $\begin{array}{r}65 \\
100 \\
50\end{array}$ & $\begin{array}{l}710 \\
400 \\
490 \\
520 \\
610 \\
670 \\
550 \\
490 \\
780 \\
475\end{array}$ & $\begin{array}{l}300 \\
375 \\
450 \\
380 \\
450 \\
330 \\
400 \\
220 \\
385 \\
950\end{array}$ & $\begin{array}{l}960 \\
570 \\
510 \\
490 \\
410 \\
650 \\
650 \\
490 \\
820 \\
656\end{array}$ & $\begin{array}{l}\mathbf{5 4 5} \\
485 \\
384 \\
330 \\
550 \\
350 \\
600 \\
370 \\
360 \\
960\end{array}$ & $\begin{array}{l}870 \\
423 \\
530\end{array}$ & $\begin{array}{l}385 \\
338 \\
528\end{array}$ \\
\hline \multirow[t]{3}{*}{ Mean } & 17.2 & 71 & 18 & 80 & 22.3 & 570 & 425 & 620 & 493 & 604 & 417 \\
\hline & \multicolumn{5}{|c|}{$\begin{array}{l}\text { Intraesophageal end expiratory pressure } \\
(\mathrm{cm} . \mathrm{H}: 0)\end{array}$} & \multicolumn{6}{|c|}{$\begin{array}{l}\text { Compliance } \\
\left(L . / \mathrm{cm} . \mathrm{H}_{2} \mathrm{O}\right)\end{array}$} \\
\hline & \multicolumn{2}{|c|}{ Supine } & \multicolumn{2}{|c|}{ Sitting } & Prone & \multicolumn{2}{|c|}{ Supine } & \multicolumn{2}{|c|}{ Sitting } & \multicolumn{2}{|c|}{ Prone } \\
\hline Patient & SR & FR & SR & FR & FR & SR & FR & SR & FR & SR & FR \\
\hline $\begin{array}{l}\text { R. W. } \\
\text { A. DeW. } \\
\text { E. J. } \\
\text { B. B. } \\
\text { W. F. } \\
\text { G. A. } \\
\text { H. P. (I)* } \\
\text { H.P. (II)† } \\
\text { M. G. } \\
\text { J. Q. }\end{array}$ & $\begin{array}{l}+1.5 \\
+1.5 \\
-1.0 \\
-2.0 \\
-0.8 \\
+1.5 \\
-5.5 \\
-2.5 \\
+0.6 \\
-2.0\end{array}$ & $\begin{array}{l}-2.0 \\
+5.5 \\
+1.0 \\
-6.2 \\
+4.0 \\
+1.0 \\
-6.7 \\
-4.5 \\
-2.0 \\
+2.0\end{array}$ & $\begin{array}{l}-2.4 \\
+\quad 3.0 \\
=5.5 \\
=3.2 \\
=9.0 \\
=1.5 \\
=5.0 \\
=6.0 \\
=1.5 \\
-10.0\end{array}$ & $\begin{array}{l}-5.5 \\
+12.0 \\
-5.7 \\
=8.5 \\
=7.8 \\
=2.5 \\
=4.5 \\
-6.0 \\
=1.2 \\
-8.0\end{array}$ & $\begin{array}{l}-3.8-5.5 \\
+1.0+5.1 \\
-5.0-5.3\end{array}$ & $\begin{array}{l}0.110 \\
0.052 \\
0.058 \\
0.045 \\
0.145 \\
0.105 \\
0.165 \\
0.090 \\
0.107 \\
0.076\end{array}$ & $\begin{array}{l}0.068 \\
0.025 \\
0.057 \\
0.029 \\
0.080 \\
0.084 \\
0.133 \\
0.047 \\
0.058 \\
0.070\end{array}$ & $\begin{array}{l}0.150 \\
0.083 \\
0.060 \\
0.060 \\
0.086 \\
0.142 \\
0.202 \\
0.125 \\
0.136 \\
0.107\end{array}$ & $\begin{array}{l}0.130 \\
0.021 \\
0.056 \\
0.031 \\
0.104 \\
0.141 \\
0.224 \\
0.075 \\
0.055 \\
0.118\end{array}$ & $\begin{array}{l}0.130 \\
0.092 \\
0.068\end{array}$ & $\begin{array}{l}0.100 \\
0.024 \\
0.056\end{array}$ \\
\hline \multirow[t]{3}{*}{ Mean } & -0.9 & -0.8 & -4.1 & -3.8 & $-2.6-1.9$ & 0.095 & 0.065 & 0.115 & 0.096 & 0.097 & 0.060 \\
\hline & \multicolumn{5}{|c|}{$\begin{array}{l}\text { Mean mechanical resistance during inspiration } \\
(\mathrm{cm} . \mathrm{H} \mathrm{O} / \mathrm{L} . / \mathrm{sec} .)\end{array}$} & \multicolumn{6}{|c|}{$\begin{array}{c}\text { Mean mechanical resistance during expiration } \\
(\mathrm{cm} . \mathrm{H}=\mathrm{O} / L . / \mathrm{sec} .)\end{array}$} \\
\hline & \multicolumn{2}{|c|}{ Supine } & \multicolumn{2}{|c|}{ Sitting } & Prone & \multicolumn{2}{|c|}{ Supine } & \multicolumn{2}{|c|}{ Sitting } & \multicolumn{2}{|c|}{ Prone } \\
\hline Patient & SR & FR & SR & FR & FR & SR & FR & SR & FR & SR & FR \\
\hline $\begin{array}{l}\text { R. W. } \\
\text { A. DeW. } \\
\text { E. J. } \\
\text { B. B. } \\
\text { W. F. } \\
\text { G. A. } \\
\text { H. P. (I)* } \\
\text { H. P. (II)† } \\
\text { M. G. } \\
\text { J. Q. }\end{array}$ & $\begin{array}{r}7.25 \\
12.20 \\
6.80 \\
13.00 \\
3.18 \\
5.70 \\
3.25 \\
7.20 \\
10.90 \\
6.70\end{array}$ & $\begin{array}{r}6.80 \\
6.50 \\
8.90 \\
12.50 \\
2.41 \\
3.10 \\
3.65 \\
5.70 \\
8.90 \\
3.70\end{array}$ & $\begin{array}{r}4.50 \\
10.30 \\
5.20 \\
9.20 \\
2.20 \\
1.75 \\
2.80 \\
3.90 \\
12.40 \\
5.55\end{array}$ & $\begin{array}{l}5.40 \\
8.80 \\
3.60 \\
8.90 \\
3.14 \\
1.84 \\
1.74 \\
3.70 \\
9.50 \\
3.10\end{array}$ & $\begin{array}{rr}6.35 & 7.90 \\
22.80 & 10.10 \\
4.70 & 4.90\end{array}$ & $\begin{array}{r}7.25 \\
19.30 \\
10.65 \\
13.60 \\
6.80 \\
10.60 \\
4.25 \\
9.65 \\
21.80 \\
9.00\end{array}$ & $\begin{array}{r}6.90 \\
9.80 \\
9.90 \\
17.00 \\
4.46 \\
5.75 \\
3.30 \\
8.45 \\
12.00 \\
4.25\end{array}$ & $\begin{array}{r}6.80 \\
12.50 \\
8.40 \\
8.00 \\
3.45 \\
2.06 \\
2.50 \\
5.65 \\
17.50 \\
7.90\end{array}$ & $\begin{array}{r}5.30 \\
12.10 \\
5.80 \\
9.70 \\
3.27 \\
2.63 \\
1.62 \\
4.40 \\
17.00 \\
4.10\end{array}$ & $\begin{array}{r}6.40 \\
14.00 \\
6.15\end{array}$ & $\begin{array}{r}8.30 \\
10.10 \\
7.30\end{array}$ \\
\hline Mean & 7.62 & 6.22 & 5.78 & 4.97 & 11.30 & 11.29 & 8.18 & 7.48 & 6.59 & 8.82 & 8.57 \\
\hline
\end{tabular}

* First study.

† Second study-one month later. 
TABLE IV

Mechanics of breathing: Results in five patients in supine, sitting and head down positions during slow and fast respiratory rates

\begin{tabular}{|c|c|c|c|c|c|c|c|c|c|c|c|c|c|c|c|}
\hline \multirow[b]{3}{*}{ Patient } & \multicolumn{7}{|c|}{ Respiratory rate } & & \multicolumn{7}{|c|}{$\begin{array}{c}\text { Tidal volume } \\
(m l .)\end{array}$} \\
\hline & \multicolumn{2}{|c|}{ Supine } & \multicolumn{2}{|c|}{ Sitting } & & \multicolumn{2}{|c|}{ Head down } & & \multicolumn{2}{|c|}{ Supine } & & \multicolumn{2}{|c|}{ Sitting } & \multicolumn{2}{|c|}{ Head down } \\
\hline & $\overline{\mathrm{SR}}$ & $\overline{F R}$ & $\overline{S R}$ & FR & & SR & FR & & $\mathrm{SR}$ & $\overline{F R}$ & & $\mathrm{SR}$ & $\overline{F R}$ & SR & $F R$ \\
\hline $\begin{array}{l}\text { M. S. } \\
\text { M. N. (II) } \\
\text { H.P. (II) } \\
\text { M. G. } \\
\text { J.Q. }\end{array}$ & $\begin{array}{l}23 \\
18 \\
22 \\
11 \\
10\end{array}$ & $\begin{array}{r}125 \\
95 \\
100 \\
55 \\
65\end{array}$ & $\begin{array}{ll}24 & 1 \\
16 & \\
20 & 1 \\
11 & \\
15 & \end{array}$ & $\begin{array}{r}185 \\
80 \\
115 \\
79 \\
60\end{array}$ & & $\begin{array}{l}20 \\
20 \\
24 \\
15 \\
10\end{array}$ & $\begin{array}{r}150 \\
100 \\
80 \\
83 \\
70\end{array}$ & & $\begin{array}{l}460 \\
730 \\
490 \\
780 \\
475\end{array}$ & $\begin{array}{l}900 \\
325 \\
220 \\
385 \\
950\end{array}$ & & $\begin{array}{l}450 \\
867 \\
490 \\
820 \\
655\end{array}$ & $\begin{array}{l}870 \\
454 \\
370 \\
360 \\
960\end{array}$ & $\begin{array}{r}520 \\
650 \\
585 \\
1,000 \\
730\end{array}$ & $\begin{array}{r}685 \\
325 \\
330 \\
320 \\
1,130\end{array}$ \\
\hline \multirow[t]{3}{*}{ Mean } & 16.8 & 88 & $\begin{array}{ll}17.2 & 1\end{array}$ & 104 & & 17.8 & 96.6 & & 587 & 556 & & 656 & 603 & 698 & 558 \\
\hline & \multicolumn{8}{|c|}{$\begin{array}{l}\text { Intraesophageal end expiratory pressure } \\
\qquad\left(\mathrm{cm} . \mathrm{H}_{2} \mathrm{O}\right)\end{array}$} & \multicolumn{7}{|c|}{$\begin{array}{l}\text { Compliance } \\
\left(\mathrm{L} . / \mathrm{cm} . \mathrm{H}_{2} \mathrm{O}\right)\end{array}$} \\
\hline & \multicolumn{2}{|c|}{ Supine } & \multicolumn{3}{|c|}{ Sitting } & & \multicolumn{2}{|c|}{ Head down } & & \multicolumn{2}{|c|}{ Supine } & \multicolumn{2}{|r|}{ Sitting } & \multicolumn{2}{|c|}{ Head down } \\
\hline Patient & $\mathrm{SR}$ & FR & SR & & FR & & SR & FR & & $\mathrm{SR}$ & FR & $\mathrm{SR}$ & FR & SR & FR \\
\hline $\begin{array}{l}\text { M. S. } \\
\text { M. N. (II) } \\
\text { H.P. (II) } \\
\text { M. G. } \\
\text { J.Q. }\end{array}$ & $\begin{array}{l}+0.25 \\
+0.20 \\
-2.50 \\
+0.60 \\
-2.00\end{array}$ & $\begin{array}{l}+3.00 \\
-0.75 \\
-4.50 \\
-2.00 \\
+2.00\end{array}$ & $\begin{array}{l}-6 \\
-1 \\
-6 \\
-1 \\
-10\end{array}$ & $\begin{array}{l}5.00 \\
.75 \\
.00 \\
.50 \\
.00\end{array}$ & $\begin{array}{l}-2.50 \\
0 \\
-6.00 \\
-1.20 \\
-8.00\end{array}$ & & $\begin{array}{l}+0.50 \\
+1.40 \\
+1.00 \\
+1.20 \\
+1.50\end{array}$ & $\begin{array}{c}+1.50 \\
+1.10 \\
0 \\
-1.20 \\
+6.00\end{array}$ & & $\begin{array}{l}0.118 \\
0.111 \\
0.090 \\
0.107 \\
0.076\end{array}$ & $\begin{array}{l}0.065 \\
0.040 \\
0.047 \\
0.058 \\
0.070\end{array}$ & $\begin{array}{l}0.15 \\
0.18 \\
0.12 \\
0.13 \\
0.10\end{array}$ & $\begin{array}{ll}96 & 0.066 \\
86 & 0.062 \\
25 & 0.075 \\
36 & 0.055 \\
07 & 0.118\end{array}$ & $\begin{array}{l}0.231 \\
0.105 \\
0.095 \\
0.103 \\
0.086\end{array}$ & $\begin{array}{l}0.050 \\
0.040 \\
0.052 \\
0.041 \\
0.062\end{array}$ \\
\hline \multirow[t]{3}{*}{ Mean } & -0.70 & -0.45 & -5 & 5.00 & -3.50 & & +0.70 & +1.50 & & 0.100 & 0.056 & 0.15 & $50 \quad 0.075$ & 0.124 & 0.049 \\
\hline & \multicolumn{7}{|c|}{$\begin{array}{l}\text { Mean mechanical resistance during inspiration } \\
\left(\mathrm{cm} . \mathrm{H}_{2} \mathrm{O} / L . / \mathrm{sec} .\right)\end{array}$} & & \multicolumn{7}{|c|}{$\begin{array}{l}\text { Mean mechanical resistance during expiration } \\
\left(\mathrm{cm} . \mathrm{H}_{2} \mathrm{O} / \mathrm{L} . / \mathrm{sec} .\right)\end{array}$} \\
\hline & \multicolumn{2}{|c|}{ Supine } & \multicolumn{2}{|c|}{ Sitting } & & \multicolumn{2}{|c|}{ Head down } & & \multicolumn{2}{|c|}{ Supine } & & \multicolumn{2}{|c|}{ Sitting } & \multicolumn{2}{|c|}{ Head down } \\
\hline Patient & SR & $\overline{F R}$ & SR & FR & & $\mathrm{SR}$ & FR & & SR & FR & & SR & FR & SR & FR \\
\hline $\begin{array}{l}\text { M. S. } \\
\text { M. N. (II) } \\
\text { H. P. (II) } \\
\text { M. G. } \\
\text { J. Q. }\end{array}$ & $\begin{array}{r}4.20 \\
6.60 \\
7.20 \\
10.90 \\
6.70\end{array}$ & $\begin{array}{l}3.10 \\
5.65 \\
5.70 \\
8.70 \\
3.70\end{array}$ & $\begin{array}{r}2.20 \\
6.70 \\
3.90 \\
12.40 \\
5.55\end{array}$ & $\begin{array}{l}2.16 \\
3.45 \\
3.70 \\
9.50 \\
3.10\end{array}$ & & $\begin{array}{l}2.95 \\
7.80 \\
4.35 \\
9.90 \\
5.35\end{array}$ & $\begin{array}{l}1.75 \\
4.50 \\
6.20 \\
8.40 \\
4.00\end{array}$ & & $\begin{array}{r}3.85 \\
9.50 \\
9.65 \\
21.80 \\
9.00\end{array}$ & $\begin{array}{r}5.60 \\
7.75 \\
8.45 \\
12.00 \\
4.2\end{array}$ & & $\begin{array}{r}2.10 \\
6.00 \\
5.65 \\
17.50 \\
7.90\end{array}$ & $\begin{array}{r}2.25 \\
7.23 \\
4.40 \\
17.00 \\
4.10\end{array}$ & $\begin{array}{r}2.20 \\
8.50 \\
6.55 \\
18.40 \\
6.70\end{array}$ & $\begin{array}{r}2.15 \\
7.80 \\
6.85 \\
10.50 \\
4.70\end{array}$ \\
\hline Mean & 7.09 & 5.36 & 6.14 & 4.38 & & 6.07 & 4.97 & & 10.76 & 7.61 & & 7.83 & 7.00 & 8.47 & 6.40 \\
\hline
\end{tabular}

variety of diseases, the response to positional changes is essentially the same as in the chronic pulmonary emphysema group. However, some interesting differences have to be pointed out. While in chronic pulmonary emphysema, a change in respiratory rate produces a significant drop of compliance in all positions, in this group it occurs only in the supine position. In addition, there is a significant difference in inspiratory resistance at different respiratory rates in the supine position, and in expiratory resistance in the supine and sitting positions. The results of the prone position in this group are not suitable for statistical analysis because of the small number of subjects.

\section{Head down position}

The five patients studied in the head down position (lying on the back at an angle of approxi- mately 20 degrees) were selected from both patient groups. Compared with the supine position, the end expiratory pressure is found increased in all ; with one exception, compliance is essentially equal and the resistance values are somewhat lower. However, only the difference in expiratory resistance is statistically significant. There is a significant decrease of compliance with increase in the respiratory rate in the head down position. Our results do not confirm the claim that the head down position is an important method for improving the ventilation in chronic pulmonary emphysema (7).

\section{Lateral position}

The six patients of this group were all expected to show some unilateral dysfunction. It is realized, however, that the latter could not be quan- 
titatively evaluated without bronchospirometry. This does not invalidate the results which are interpreted on the assumption that intraesophageal pressure differences express the result of the intrapleural pressure differences on both sides. If the diseased side lies below, the end expiratory pressure is higher than if the healthy side lies below; the values for the supine position are usually somewhere between the two. In the four cases where simultaneous intrapleural and intraesophageal pressures were taken, the intrapleural compliance tended to be somewhat lower than the intraesophageal compliance if the patient was lying on the diseased lung and considerably higher if he was lying on the healthy side. However, the direction of change was the same for intrapleural and intraesophageal measurements. It must be kept in mind that during inspiration the hydrostatic pressure of a pleural effusion tends to fall because of the increase in thorax volume, thereby increasing the total pressure difference. This would lead to an apparent decrease of compliance, more marked in intrapleural measurements than in intraesophageal measurements, while mechanical resistance would increase. This is probably a minor factor in the supine position, where the differences in intrapleural and intraesophageal compliance are small. Both intrapleural and intraesophageal compliances are higher when the healthy lung is below as compared to the diseased lung below. There is no significant change in compliance with change in the respiratory rate. Mechanical resistance is higher during expiration than during inspiration in both lateral positions, but the differences are less marked when the healthy lung is below. Both inspiratory and expiratory resistance are lower in the healthy side down as compared to the diseased side down posi-

TABLE $V$

Mechanics of breathing: Results of six patients in supine, sitting and two lateral positions during slow and fast respiratory rates

\begin{tabular}{|c|c|c|c|c|c|c|c|c|c|c|c|c|c|c|c|c|}
\hline \multirow[b]{3}{*}{ Patient } & \multicolumn{8}{|c|}{ Respiratory rate } & \multicolumn{8}{|c|}{$\begin{array}{c}\text { Tidal volume } \\
(\mathrm{ml} .)\end{array}$} \\
\hline & \multicolumn{2}{|c|}{ Supine } & \multicolumn{2}{|c|}{ Sitting } & \multicolumn{2}{|c|}{ D. Lat.* } & \multicolumn{2}{|c|}{ H. Lat. } & \multicolumn{2}{|c|}{ Supine } & \multicolumn{2}{|c|}{ Sitting } & \multicolumn{2}{|c|}{ D. Lat.* } & \multicolumn{2}{|c|}{ H. Lat. $\uparrow$} \\
\hline & $\overline{\text { SR }}$ & $\overline{\text { FR }}$ & SR & $\overline{F R}$ & SR & $\overline{F R}$ & SR F & $\overline{F R}$ & SR & $\overline{F R}$ & SR & $\overline{F R}$ & $\overline{S R}$ & $\mathbf{F R}$ & SR & $\overline{F R}$ \\
\hline $\begin{array}{l}\text { J. C. } \\
\text { W. F. } \\
\text { B. B. } \\
\text { H. T. } \\
\text { G. A. } \\
\text { A. A. }\end{array}$ & $\begin{array}{l}20 \\
16 \\
20 \\
11 \\
15 \\
24\end{array}$ & $\begin{array}{r}100 \\
65 \\
50 \\
80 \\
83 \\
60\end{array}$ & $\begin{array}{r}6 \\
17 \\
30 \\
14 \\
26\end{array}$ & $\begin{array}{r}100 \\
71 \\
70 \\
80 \\
100\end{array}$ & $\begin{array}{l}22 \\
15 \\
22 \\
14 \\
23 \\
24\end{array}$ & $\begin{array}{l}65 \\
72 \\
55 \\
80 \\
78 \\
50\end{array}$ & $\begin{array}{l}24 \\
16 \\
25 \\
11 \\
23 \\
24\end{array}$ & $\begin{array}{l}60 \\
75 \\
50 \\
80 \\
86 \\
55\end{array}$ & $\begin{array}{l}560 \\
610 \\
520 \\
850 \\
670 \\
560\end{array}$ & $\begin{array}{l}245 \\
450 \\
380 \\
350 \\
330 \\
500\end{array}$ & $\begin{array}{l}592 \\
410 \\
490 \\
750 \\
650\end{array}$ & $\begin{array}{l}270 \\
550 \\
330 \\
435 \\
\mathbf{3 5 0}\end{array}$ & $\begin{array}{l}\mathbf{5 3 0} \\
\mathbf{5 5 0} \\
\mathbf{5 0 0} \\
\mathbf{7 5 0} \\
\mathbf{6 3 0} \\
\mathbf{3 9 0}\end{array}$ & $\begin{array}{l}\mathbf{3 8 5} \\
\mathbf{6 0 0} \\
\mathbf{3 8 5} \\
\mathbf{5 5 0} \\
\mathbf{3 9 0} \\
\mathbf{3 0 0}\end{array}$ & $\begin{array}{l}490 \\
600 \\
500 \\
870 \\
670 \\
578\end{array}$ & $\begin{array}{l}440 \\
470 \\
430 \\
470 \\
330 \\
390\end{array}$ \\
\hline \multirow[t]{3}{*}{ Mean } & 17.7 & 73 & 18.6 & 80.4 & 20 & 66.6 & 20.5 & 67.6 & 628 & 376 & 578 & 387 & 558 & 435 & 618 & 422 \\
\hline & \multicolumn{8}{|c|}{$\begin{array}{l}\text { Intraesophageal end expiratory pressure } \\
\left(\mathrm{cm} . \mathrm{H}_{2} \mathrm{O}\right)\end{array}$} & \multicolumn{8}{|c|}{$\begin{array}{l}\text { Compliance } \\
\left(L . / \mathrm{cm} . \mathrm{H}_{2} O\right)\end{array}$} \\
\hline & \multicolumn{2}{|c|}{ Supine } & \multicolumn{2}{|c|}{ Sitting } & \multicolumn{2}{|c|}{ D. Lat.* } & \multicolumn{2}{|c|}{ H. Lat. } & \multicolumn{2}{|c|}{ Supine } & \multicolumn{2}{|c|}{ Sitting } & \multicolumn{2}{|c|}{ D. Lat.* } & \multicolumn{2}{|c|}{ H. Lat. $\dagger$} \\
\hline Patient & SR & FR & SR & FR & SR & $\overline{F R}$ & SR & FR & SR & FR & SR & $\mathbf{F R}$ & SR & FR & SR & $\overline{\text { FR }}$ \\
\hline $\begin{array}{l}\text { J. C. } \\
\text { W. } \\
\text { B. B. } \\
\text { H. } \\
\text { T. } \\
\text { G. A. } \\
\text { A. A. }\end{array}$ & $\begin{array}{c}+1.8 \\
-0.8 \\
-2.0 \\
0 \\
+1.5 \\
+2.5\end{array}$ & $\begin{array}{l}+0.9 \\
+4.0 \\
-6.2 \\
-1.2 \\
+1.0 \\
+3.0\end{array}$ & $\begin{array}{l}+1.1 \\
-9.0 \\
-3.2 \\
-5.0 \\
-1.5\end{array}$ & $\begin{array}{l}-2.0 \\
-7.8 \\
-8.5 \\
-5.0 \\
-2.5\end{array}$ & $\begin{array}{l}+1.0 \\
-1.3 \\
-0.5 \\
-1.0 \\
+4.5 \\
+2.0\end{array}$ & $\begin{array}{l}-0.5 \\
-0.3 \\
-3.0 \\
-0.5 \\
+1.0 \\
+0.5\end{array}$ & $\begin{array}{l}-0.7 \\
-3.5 \\
-4.0 \\
-1.5 \\
+2.5 \\
+2.0\end{array}$ & $\begin{array}{ll}7 & -1.0 \\
5 & -2.0 \\
0 & -6.0 \\
5 & +0.5 \\
5 & -2.0 \\
0 & +0.5\end{array}$ & $\begin{array}{l}0.143 \\
0.145 \\
0.045 \\
0.112 \\
0.105 \\
0.087\end{array}$ & $\begin{array}{l}0.162 \\
0.080 \\
0.028 \\
0.112 \\
0.084 \\
0.072\end{array}$ & $\begin{array}{l}0.161 \\
0.086 \\
0.060 \\
0.200 \\
0.142\end{array}$ & $\begin{array}{l}0.161 \\
0.104 \\
0.031 \\
0.210 \\
0.141\end{array}$ & $\begin{array}{l}0.115 \\
0.143 \\
0.060 \\
0.120 \\
0.064 \\
0.050\end{array}$ & $\begin{array}{l}0.136 \\
0.150 \\
0.031 \\
0.127 \\
0.050 \\
0.038\end{array}$ & $\begin{array}{l}0.205 \\
0.135 \\
0.056 \\
0.160 \\
0.080 \\
0.090\end{array}$ & $\begin{array}{l}0.220 \\
0.095 \\
0.034 \\
0.160 \\
0.074 \\
0.072\end{array}$ \\
\hline \multirow[t]{3}{*}{ Mean } & +0.5 & +0.5 & -3.5 & -5.2 & +0.8 & -0.5 & -0.9 & $9-2.2$ & 0.106 & 0.090 & 0.130 & 0.130 & 0.092 & 0.089 & 0.121 & 0.109 \\
\hline & \multicolumn{8}{|c|}{$\begin{array}{l}\text { Mean mechanical resistance during inspiration } \\
\left(\mathrm{cm} . \mathrm{H}_{2} \mathrm{O} / \mathrm{L} . / \mathrm{sec} .\right)\end{array}$} & \multicolumn{8}{|c|}{$\begin{array}{l}\text { Mean mechanical resistance during expiration } \\
\left(\mathrm{cm} . \mathrm{H}_{2} \mathrm{O} / L . / \mathrm{sec} .\right)\end{array}$} \\
\hline & \multicolumn{2}{|c|}{ Supine } & \multicolumn{2}{|c|}{ Sitting } & \multicolumn{2}{|c|}{ D. Lat.* } & \multicolumn{2}{|c|}{ H. Lat. $\dagger$} & \multicolumn{2}{|c|}{ Supine } & \multicolumn{2}{|c|}{ Sitting } & \multicolumn{2}{|c|}{ D. Lat.* } & \multicolumn{2}{|c|}{ H. Lat. $\dagger$} \\
\hline Patient & SR & FR & $\overline{\text { SR }}$ & $\overline{F R}$ & SR & $\overline{F R}$ & SR & FR & SR & FR & SR & FR & SR & FR & SR & FR \\
\hline $\begin{array}{l}\text { J. C. } \\
\text { W. F. } \\
\text { B. B. } \\
\text { H. T. } \\
\text { G. A. } \\
\text { A. A. }\end{array}$ & $\begin{array}{r}2.18 \\
3.18 \\
13.00 \\
1.50 \\
5.70 \\
4.75\end{array}$ & $\begin{array}{r}2.83 \\
2.41 \\
12.50 \\
2.40 \\
3.10 \\
5.25\end{array}$ & $\begin{array}{l}2.34 \\
2.20 \\
9.20 \\
1.65 \\
1.75\end{array}$ & $\begin{array}{l}.60 \\
3.14 \\
8.90 \\
1.00 \\
1.84\end{array}$ & $\begin{array}{r}4.10 \\
2.30 \\
10.80 \\
1.00 \\
3.30 \\
10.30\end{array}$ & $\begin{array}{l}3.40 \\
2.48 \\
8.75 \\
1.65 \\
3.90 \\
6.85\end{array}$ & $\begin{array}{r}2.00 \\
1.68 \\
11.20 \\
2.36 \\
4.30 \\
5.60\end{array}$ & $\begin{array}{r}3.15 \\
5.65 \\
10.75 \\
1.70 \\
3.90 \\
4.30\end{array}$ & $\begin{array}{r}2.38 \\
6.80 \\
13.60 \\
2.50 \\
10.60 \\
8.80\end{array}$ & $\begin{array}{r}2.80 \\
4.46 \\
17.00 \\
3.00 \\
\mathbf{5 . 7 5} \\
\mathbf{5 . 5 0}\end{array}$ & $\begin{array}{l}2.32 \\
3.45 \\
8.00 \\
2.70 \\
2.06\end{array}$ & $\begin{array}{l}2.22 \\
3.27 \\
9.70 \\
1.30 \\
2.63\end{array}$ & $\begin{array}{r}3.75 \\
5.30 \\
16.60 \\
2.00 \\
11.10 \\
10.00\end{array}$ & $\begin{array}{r}2.85 \\
3.93 \\
18.20 \\
2.35 \\
5.10 \\
8.90\end{array}$ & $\begin{array}{r}2.22 \\
6.05 \\
18.40 \\
2.72 \\
5.20 \\
4.95\end{array}$ & $\begin{array}{r}3.21 \\
8.65 \\
19.40 \\
2.27 \\
3.84 \\
3.00\end{array}$ \\
\hline Mean & 5.05 & 4.75 & 3.43 & 3.50 & 5.30 & 4.48 & 4.53 & 4.81 & 7.45 & 6.42 & 3.70 & 3.80 & 8.12 & 6.88 & 6.60 & 6.72 \\
\hline
\end{tabular}

* Lateral position: Lying on diseased side.
t Lateral position: Lying on healthy side. 


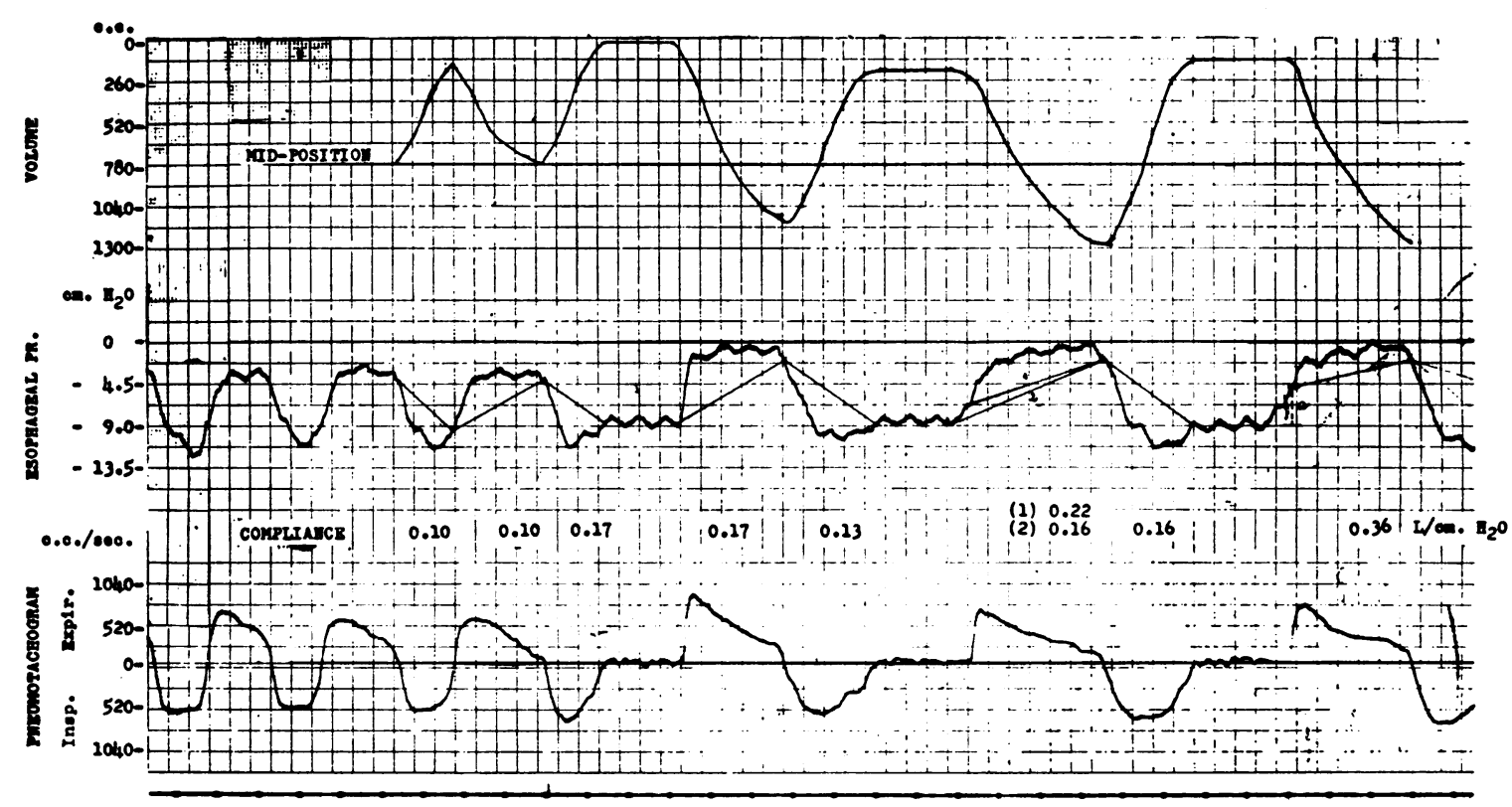

FIGURe 1

Change in compliance due to unequal distribution of air (sitting position with inspiratory pause and change of mid-position with respiratory rate). Change of end inspiratory pressure during the pause with transmission of heart beats. At a, glottis closure and change of intraesophageal pressure due to action of expiratory muscles with zero air flow.

tion. However, the scatter of the individual scores is quite large and the differences are not statistically significant as compared to the supine position.

\section{DISCUSSION}

The overall changes in the mechanics of breathing in the supine, sitting and prone positions for both groups of patients are essentially the same as for the normal group previously described.

There is, however, one essential difference between the two patient groups and the normal group. The normal subjects do not show any change in compliance or resistance with an increase in the respiratory rate. The chronic pulmonary emphysema group shows a marked drop of compliance with an increase in rate in all positions, while the resistance values remain essentially the same. The group with various cardiopulmonary diseases reveals a significant drop of compliance in the supine and prone positions, a significant drop of inspiratory resistance in the supine and of expiratory resistance in the sitting and supine positions. The dependence of compliance upon respiratory rate is shown in Figure
1 where compliance decreases with an increase of respiratory rate. This is usually explained on the basis of unequal ventilation $(6,8,9)$. During increased respiratory rates, a smaller total volume change occurs per given pressure. In view of the fact that under these conditions a time element influences the compliance, the latter is no longer a pure expression of the elastic properties alone if measured by the described method. In an elastic system, the response to a force depends only on the magnitude of the force applied while in a viscous system, the response depends on the duration of the time during which the force is applied (10). According to Mount (11), part of the elastic element of the lungs is in series with a variable tissue deformation resistance which confers time dependency upon the elastic properties of the lung. The changes of compliance with different respiratory rates and different body positions, are shown together with the results of the seven-minute nitrogen washout in the supine (Figure 2) and in the supine and sitting (Figure 3 ) positions.

It will be seen that, in general, low compliance values go together with high intrapulmonary mixing indices. Inasmuch as most of these patients 
tend to have an increased respiratory rate even under basal conditions, it is conceivable that the "basal" compliance which we obtain is already reduced and would be considerably higher if measured under static conditions. Furthermore, the nitrogen washout method tends to give too favorable a picture in hyperventilating subjects. These two factors could explain why this correlation is not perfect.

From our results it would seem that the supine, head down and lateral positions require more effort for the breathing process than the sitting and prone positions. They also are accompanied by poorer intrapulmonary mixing. Such changes become important in the patient with pulmonary insufficiency where minimal additional efforts may suffice to produce a severe and embarrassing picture $\left(\mathrm{CO}_{2}\right.$ narcosis, atelectasis, drug addiction, etc.). The striking relief of dyspnea which can be observed in many patients assuming the sitting position may also be explained by the improved mechanics of breathing noted in this position.

\section{SUMMARY}

1. Studies in the mechanics of breathing in a group of patients with chronic pulmonary emphysema and a group of patients with various cardiopulmonary diseases are reported. Compliance and mechanical resistance were measured during slow and fast respiratory rates in the supine, sitting, prone, head down and lateral positions.

2. Both patient groups showed lower mean compliance and considerably higher mean mechanical resistance than the normal group. Expiratory resistance was considerably higher than inspiratory resistance in both patient groups.

3. The positional changes were in the same direction as noted in the normal group. Compliance was lowest in the supine and highest in the sitting

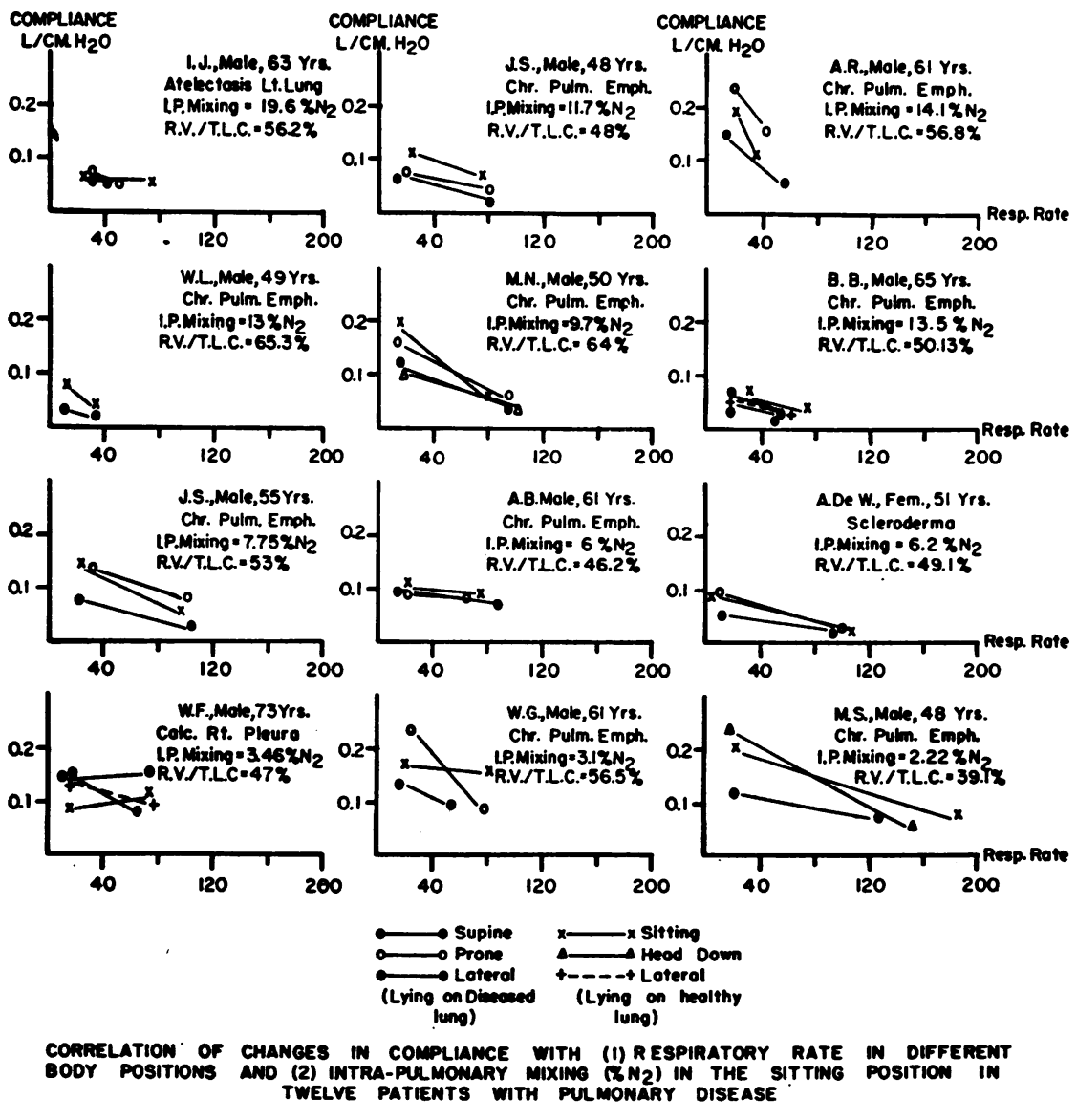

Figure 2 


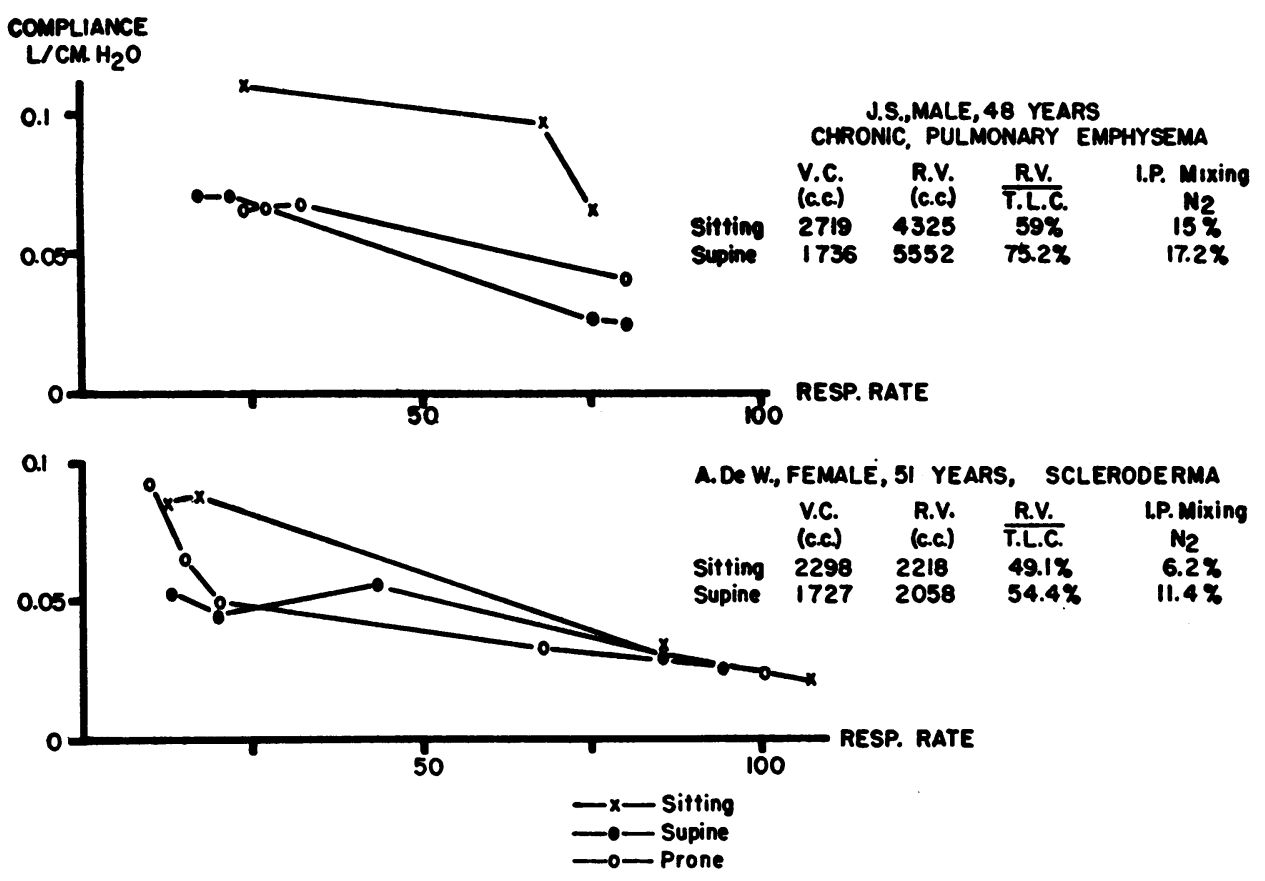

RELATIONSHIPS OF RESIDUAL VOLUME (R.V.) ANO INTRA-PULMONARY MIXING (I.P.MOXMG XN/2) WITH CHANGE IN COMPLIANCE DURING INCREASING RESPIRATORY RATE IN DIFFERENT BODY POSITIONS IN TWO PATIENTS WITH PULMONARY DISEASE.

Figure 3

position and mechanical resistance was highest in the supine and lowest in the sitting position. The prone position compared favorably with the sitting position while the head down and lateral were nearer the supine position.

4. Patients with predominantly unilateral disease showed in general a lower compliance and higher resistance when lying on the diseased side as compared to lying on the healthy side.

5. In both patient groups there was a marked drop of compliance with increase in the respiratory rate in all positions studied. The drop in compliance was smallest in the sitting position.

\section{REFERENCES}

1. Attinger, E. O., Monroe, R. G., and Segal, M. S., The mechanics of breathing in different body positions. I. In normal subjects. J. Clin. Invest., 1956, 35, 904.

2. von Neergard, K., and Wirz, K., Die Messung der Strómungswiderstände in den Atemwegen des Men- schen, insbesondere bei Rothima und Emphysem. Ztschr. Klin. Med., 1927, 105, 51.

3. Dayman, H., Mechanics of airflow in health and in emphysema. J. Clin. Invest., 1951, 30, 1175.

4. Dayman, H. G., Lung distention and morphology. Clin. Res. Proc., 1955, 3, 24.

5. Fry, D. L., Ebert, R. V., Stead, W. W., and Brown, C. C., The mechanics of pulmonary ventilation in normal subjects and in patients with emphysema. Am. J. Med., 1954, 16, 80.

6. Mead, J., Lindgren, I., and Gaensler, E. A., The mechanical properties of the lungs in emphysema. J. Clin. Invest., 1955, 34, 1005.

7. Barach, A. L., and Beck, G. J., The ventilatory effects of the head-down position in pulmonary emphysema. Am. J. Med., 1954, 16, 55.

8. Fry, D. L., Personal communication.

9. Attinger, E. O., Goldstein, M. M., and Segal, M. S., Ventilation in chronic pulmonary emphysema. In press.

10. McIlroy, M. B., The physical properties of normal lungs removed after death. Thorax, 1952, 7, 285.

11. Mount, L. E., The ventilation flow-resistance and compliance of rat lungs. J. Physiol., 1955, 127, 157. 